\title{
Pulmonary arteriole gene expression signature in idiopathic pulmonary fibrosis
}

\author{
Nina M. Patel*,\#, Steven M. Kawut ${ }^{\star}$, Sanja Jelic*, Selim M. Arcasoy*,\#,, \\ David J. Lederer*,\#,+ and Alain C. Borczuk ${ }^{\S}$
}

ABSTRACT: A third of patients with idiopathic pulmonary fibrosis (IPF) develop pulmonary hypertension (PH-IPF), which is associated with increased mortality. Whether an altered gene expression profile in the pulmonary vasculature precedes the clinical onset of PH-IPF is unknown. We compared gene expression in the pulmonary vasculature of IPF patients with and without PH with controls.

Pulmonary arterioles were isolated using laser capture microdissection from 16 IPF patients: eight with PH (PH-IPF) and eight with no PH (NPH-IPF), and seven controls. Probe was prepared from extracted RNA, and hybridised to Affymetrix Hu133 2.0 Plus genechips. Biometric Research Branch array tools and Ingenuity Pathway Analysis software were used for analysis of the microarray data.

Univariate analysis revealed 255 genes that distinguished IPF arterioles from controls $(p<0.001)$. Mediators of vascular smooth muscle and endothelial cell proliferation, Wnt signalling and apoptosis were differentially expressed in IPF arterioles. Unsupervised and supervised clustering analyses revealed similar gene expression in PH-IPF and NPH-IPF arterioles.

The pulmonary arteriolar gene expression profile is similar in IPF patients with and without coexistent PH. Pathways involved in vascular proliferation and aberrant apoptosis, which may contribute to pulmonary vascular remodelling, are activated in IPF patients.

KEYWORDS: DNA microarray, laser capture microdissection, pulmonary arterial hypertension, usual interstitial pneumonia

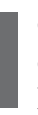
diopathic pulmonary fibrosis (IPF) is a debilitating and fatal diffuse parenchymal lung disease with a median survival of $<3$ years. More than a third of patients with IPF already have pulmonary hypertension (PH-IPF) at the time of initial evaluation for lung transplantation, with an overwhelming majority developing clinically overt $\mathrm{PH}$ by the time of transplantation [1, 2]. It is unclear if the subset of IPF patients with $\mathrm{PH}$ have a distinct vascular molecular phenotype, or whether all patients with IPF have molecular differences in pulmonary vascular gene expression compared to controls. It is valuable to discern the mechanism of pulmonary vascular dysfunction in IPF, as PHIPF is associated with decreased functional status and an increased risk of death [1,3].

Pulmonary arterioles from patients with PH-IPF exhibit marked intimal hypertrophy, medial smooth muscle cell proliferation and adventitial thickening due to fibroblast accumulation and extracellular matrix deposition [1]. Previous studies have utilised DNA microarrays to evaluate gene expression in idiopathic pulmonary arterial hypertension (PAH) and PH-IPF. Genes associated with transforming growth factor (TGF)- $\beta$, extracellular signal-related kinase (ERK)/ mitogen-activated protein kinase (MAPK), plateletderived growth factor (PDGF) and cAMP signalling are differentially expressed in whole-lung homogenates from patients with $\mathrm{PAH}$ compared with patients with PH-IPF [4]. Decreased expression of angiogenic genes and upregulation of genes associated with vascular remodelling have been observed in whole-lung homogenates in PH-IPF [5]. Considering that the pulmonary vasculature accounts for a relatively small portion of gene expression in whole-lung homogenates, differences in gene expression observed in these studies may predominantly reflect changes in the lung parenchyma rather than the pulmonary vasculature. Ascertaining directly whether gene expression is altered in the pulmonary vasculature of IPF patients with and without $\mathrm{PH}$ is clinically relevant as differences between the two groups would imply
AFFILIATIONS

*Division of Pulmonary, Allergy and Critical Care Medicine, Columbia University, New York, NY, \# Interstitial Lung Disease Program, New York Presbyterian Hospital, New York, NY,

"Dept of Medicine and the Center for Clinical Epidemiology and Biostatistics, Perelman School of Medicine, University of Pennsylvania, Philadelphia, PA,

+Lung Transplantation Program, New York Presbyterian Hospital, New York, $N Y$, and

${ }^{\S}$ Dept of Pathology and Cell Biology, Columbia University, New York, NY, USA.

CORRESPONDENCE

N.M. Patel

Division of Pulmonary, Allergy and

Critical Care Medicine

Columbia University

622 W. 168th Street

PH 8 East

Room 101

New York

NY 10032

USA

E-mail: np2199@columbia.edu

Received:

May 292012

Accepted after revision:

Aug 292012

First published online:

Sept 202012 
distinct vascular phenotypes. Differences in vascular gene expression in IPF compared with controls would suggest specific pathologic processes in IPF, which might lead to pulmonary vascular remodelling.

Thus, we assessed the gene expression of laser capture microdissected (LCM) pulmonary arterioles from IPF patients free of $\mathrm{PH}(\mathrm{NPH}-\mathrm{IPF})$ and those with coexistent $\mathrm{PH}$ and compared it with controls. We sought to identify genes and biological pathways that could potentially mediate the development of vascular remodelling in IPF. Some of the results have previously been reported as an abstract [6].

\section{METHODS}

\section{Study population}

Please see the online supplementary material for full details. We identified potentially eligible study subjects by querying the Columbia University Tissue Bank database for the terms "lung" and "usual interstitial pneumonia" (UIP) and/or "pulmonary hypertension". We included IPF patients who met American Thoracic Society criteria (previous and current) for a diagnosis of IPF [7, 8]. Patients with IPF were considered in the PH-IPF group if they had a mean pulmonary arterial pressure (mPAP) $>25 \mathrm{mmHg}$ with a pulmonary capillary wedge pressure $\leqslant 15 \mathrm{mmHg}$ measured by right heart catheterisation, meeting World Health Organization (WHO) group 3 criteria $[9,10]$. IPF patients were included in the NPH-IPF group if they had $\mathrm{mPAP} \leqslant 25 \mathrm{mmHg}$.

We excluded patients with clinical evidence of collagen vascular disease, another known aetiology for pulmonary hypertension (e.g. chronic obstructive pulmonary disease, obstructive sleep apnoea and thromboembolic disease), left ventricular dysfunction or left-sided valvular disease, missing haemodynamic or pulmonary function data and age $<18$ years.

15 IPF samples were obtained from lung explant and one from surgical lung biopsy. Specimens from four unused donor lung shavings and three normal lung sections obtained during resection of pulmonary carcinoid tumours were used as controls. Approval and a waiver of consent for clinical data collection were obtained from the Columbia University Institutional Review Board (IRB-AAAC0695).

\section{Processing and LCM of tissue samples}

All lung tissues were obtained peri-operatively, snap-frozen in liquid nitrogen and stored at $-80^{\circ} \mathrm{C}$. At the time of microdissection, the tissue samples were sliced into 5- $\mu \mathrm{m}$ sections, fixed with $95 \%$ ethanol and air-dried on PALM MembraneSlides (PALM Microlaser Technologies, Bernried, Germany). The sections were stained using the LCM Staining Kit (Ambion, Foster City, CA, USA). The Zeiss Axiovert Microscope (Carl Zeiss Microimaging Inc., Thornwood, NY, USA) with the PALM Microbeam LCM System (PALM Microlaser Technologies) was utilised for vessel dissection. All pulmonary arterioles (100-450 $\mu \mathrm{m})$ that could be visualised within a bronchovascular bundle were isolated using LCM.

\section{RNA isolation, microarray processing and analysis, and immunohistochemical staining}

The RNAqueous-Micro Kit (Ambion) was utilised to isolate RNA. The modified Eberwine procedure was used to amplify and biotinylate RNA for a minimum goal of $15 \mu \mathrm{g}$ RNA for hybridisation. Target cRNA was hybridised to Affymetrix Hu133 2.0 Plus oligonucleotide arrays (Affymetrix, Santa Clara, CA, USA). Biometric Research Branch array tools software v3.7.2. (Richard Simon and Amy Lam at the National Cancer Institute, Bethesda, MA, USA) was used for normalisation, filtering and initial quality control assessment of the genechip data. Supervised analyses of differential gene expression between class assignments was performed using univariate analyses with $\mathrm{p}<0.001$. The false discovery rate for all comparisons was set at $\mathrm{q}<10 \%$. A permutation analysis was also performed utilising 10000 random permutations, with a global $\mathrm{p}<0.01$. Gene lists were further categorised into biological pathways using Ingenuity Pathways Analysis (IPA; Ingenuity Systems, Redwood City, CA, USA).

Paraffinised tissue blocks of 10 IPF cases (five PH-IPF and five $\mathrm{NPH}-\mathrm{IPF}$ ) and five normal donor lungs were sectioned and stained for antibodies against secreted frizzled-related protein (SFRP)-2 (monoclonal rabbit antihuman) (Sigma-Aldrich Corp. St Louis, MO, USA), SPARC-related modular calcium binding (SMOC)-2 (polyclonal rabbit antihuman) (Lifespan Biosciences Inc., Seattle, WA, USA) and signal transducer and activator of transcription (STAT1) (monoclonal rabbit antihuman) (Sigma) using the DAKO Envision Dual Link kit (Dako, Glostrup, Denmark). Examination for statistical significance was performed using Fisher's exact test (SPSS, v16.0; IBM North America, New York, NY, USA).

\section{RESULTS}

We included eight patients with PH-IPF, eight with NPH-IPF and seven controls (table 1). IPF patients were older than the controls and predominantly male. Patients with PH-IPF and NPH-IPF were similar with regards to age, sex, race, smoking history and lung function. Pulmonary function data revealed the presence of a restrictive impairment and the absence of obstructive physiology in both groups. One patient had mild upper lobe emphysema by computed tomography (CT); no patients had significant emphysema histologically or by chest CT. Notably, the degree of PH present in the PH-IPF group was mild, with mPAP of $29 \mathrm{mmHg}$.

The raw gene expression values for four commonly expressed endothelial and epithelial genes were examined to confirm the isolation of pulmonary arterioles while minimising the inclusion of lung parenchyma or airways. Endothelial gene expression was elevated across all samples, while epithelial gene expression was minimal (online supplementary table E1), confirming the successful isolation of pulmonary arterioles.

We first compared PH-IPF arterioles to NPH-IPF arterioles. Unsupervised hierarchical clustering is a method of grouping gene microarray data according to similarity of content between groups. The dendogram resulting from unsupervised hierarchical clustering analysis of PH-IPF and NPH-IPF arterioles revealed no separation between the two groups (fig. 1), and there were no significant differences in gene expression by univariate analysis (online supplementary table E2).

We then analysed the gene expression of the combined IPF samples (PH-IPF + NPH-IPF) versus controls. Unsupervised hierarchical clustering demonstrated a significant separation between the IPF samples and the controls (fig. 2). 255 genes 


\begin{tabular}{|c|c|c|c|}
\hline & PH-IPF & NPH-IPF & Controls \\
\hline Subjects & 8 & 8 & 7 \\
\hline Age years & $59 \pm 5$ & $63 \pm 4$ & $45 \pm 11$ \\
\hline Female \% & 38 & 38 & 86 \\
\hline \multicolumn{4}{|l|}{ Race/ethnicity \% } \\
\hline Non-Hispanic white & 88 & 75 & 86 \\
\hline Hispanic & 12 & 25 & 14 \\
\hline Body mass index $\mathrm{kg} \cdot \mathrm{m}^{-2}$ & $27 \pm 5$ & $25 \pm 2^{\#}$ & \\
\hline Ever-smoker \% & 50 & $43^{\#}$ & \\
\hline Duration of IPF years & $3(1--4)$ & $1.5(1-4)$ & \\
\hline FVC \% pred & $55 \pm 20$ & $48 \pm 14$ & \\
\hline FVC absolute L & $2.2 \pm 1.0$ & $1.9 \pm 0.8$ & \\
\hline FEV $1 \%$ pred & $59 \pm 17$ & $54 \pm 18$ & \\
\hline FEV1 absolute $L$ & $1.8 \pm 0.6$ & $1.6 \pm 0.7$ & \\
\hline FEV $1 / F V C \%$ & $87 \pm 9$ & $81 \pm 6$ & \\
\hline TLC \% pred & $53 \pm 20$ & $48 \pm 13^{\#}$ & \\
\hline DLco \% pred & $21 \pm 10$ & $34 \pm 9$ & \\
\hline 6-min walk distance $\mathrm{m}$ & $273 \pm 75^{\circ}$ & $468 \pm 87^{+}$ & \\
\hline \multicolumn{4}{|l|}{ Haemodynamics } \\
\hline RAP $\mathrm{mmHg}$ & $3 \pm 2^{\#}$ & $1 \pm 2^{\#}$ & \\
\hline Mean PAP mmHg & $29 \pm 4$ & $12 \pm 2$ & \\
\hline PCWP mmHg & $9 \pm 3$ & $2 \pm 2$ & \\
\hline Cardiac index $L \cdot \mathrm{min}^{-1} \cdot \mathrm{m}^{-2}$ & $3.4 \pm 1.5$ & $2.4 \pm 0.5^{\#}$ & \\
\hline PVR dyn $\cdot s \cdot \mathrm{cm}^{-5}$ & $415 \pm 304$ & $214 \pm 65^{\#}$ & \\
\hline
\end{tabular}

Data are presented as $\mathrm{n}$, mean $\pm \mathrm{SD}$ or median (interquartile range). $\mathrm{PH}$ : pulmonary hypertension; IPF: idiopathic pulmonary fibrosis; NPH: no pulmonary hypertension; FVC: forced vital capacity; \% pred: \% predicted; FEV1: forced expiratory volume in $1 \mathrm{~s}$; TLC: total lung capacity; DLCO: diffusing capacity of the lung for carbon monoxide; RAP: right atrial pressure; PAP: pulmonary arterial pressure; PCWP: pulmonary capillary wedge pressure; PVR: pulmonary vascular resistance. ${ }^{\#}: n=7 ;{ }^{\bullet}: n=5 ;{ }^{+}: n=6$.

were differentially expressed in IPF arterioles as compared to control vessels using univariate analysis $(\mathrm{p}<0.001, \mathrm{q}<10 \%)$ (fig. 3), with selected results shown in table 2 (full list in online supplementary table E4). Two mediators of Wnt signalling, SFRP2 and CCND1, were upregulated in IPF arterioles, along with members of the complement family (C4A, C4B and CFH). Expression of STAT1 and SMOC2, a transcription factor and extracellular protein associated with vascular proliferation, was increased in IPF arterioles.

Conversely, members of the Kruppel-like family of transcription factors (KLF6 and 10), which modulate endothelial function, were downregulated in IPF arterioles compared with controls. S1PR1, whose ligand sphingosine 1 phosphate is an effector of endothelial cell barrier integrity, was also downregulated. Expression of arginase II, a mediator of nitric oxide signalling [11], was decreased in IPF arterioles. The effectors of apoptosis, IGFBP3 and TIMP3, were downregulated in IPF arterioles.

Lastly, we analysed the gene expression of the PH-IPF group versus controls and the NPH-IPF group versus controls. The analysis of PH-IPF versus controls revealed no statistically significant genes. Results of analysis of the NPH-IPF group versus controls are displayed in online supplementary table E3.

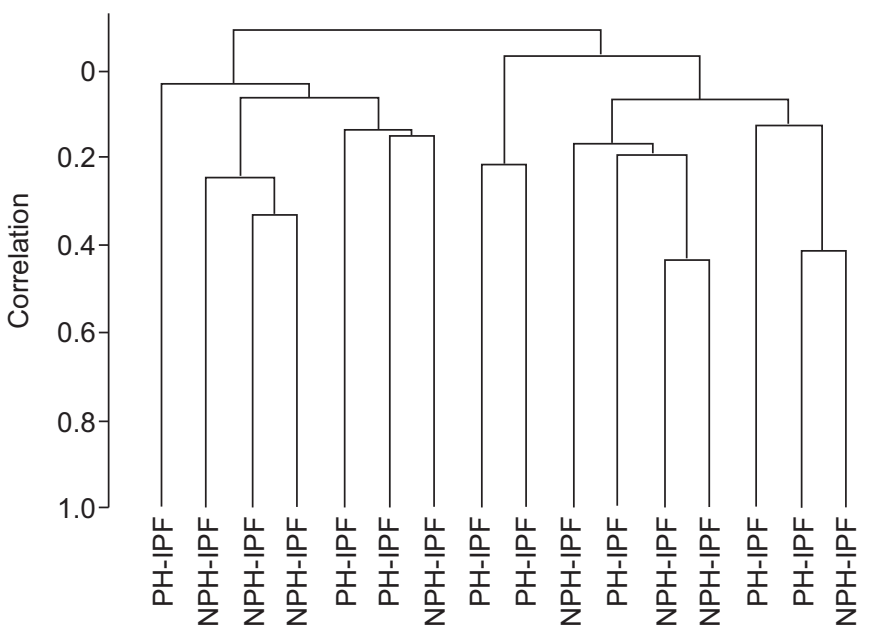

FIGURE 1. Vessels from patients with idiopathic pulmonary fibrosis (IPF) and pulmonary hypertension $(\mathrm{PH})$ versus vessels from IPF patients without $\mathrm{PH}(\mathrm{NPH}$ IPF). Unsupervised hierarchical clustering analysis using uncentered correlation and average linkage. The dendogram shows no separation of gene expression between PH-IPF and NPH-IPF samples.

\section{Biological pathways analysis}

We used IPA software to determine whether particular biological pathways and/or canonical signalling pathway gene expression was more highly represented in IPF arterioles versus controls (table 3). The top biological function for IPF arterioles was cancer, while the top molecular and cellular function was cellular growth and proliferation. The top canonical signalling pathways included amino acid metabolism, AMP kinase signalling, ciliary neutrophic factor signalling and the complement system. Genes involved in vascular endothelial growth factor (VEGF) signalling (ACTB, EIF1, PIM1 and PIK3CA) and hypoxia-related signalling (KLF10, ARG2 and ARHGAP26) were downregulated in IPF arterioles, as were markers of nitric oxide signalling (ITPR2, PIK3CA and IGFBP3). There was both up- and downregulation of genes involved in ERK/MAPK and TGF- $\beta$ signalling. STAT1 and PLAU, both mediators of smooth muscle proliferation, were upregulated in IPF arterioles. A number of genes impacting cancer and apoptosis were differentially expressed in IPF arterioles (table 3).

\section{Validation of gene array data with immunohistochemical staining}

Immunohistochemical staining of selected gene products of biological interest was performed to confirm the gene expression data (fig. 4). STAT1 and SMOC2 protein expression was significantly upregulated in IPF arterioles compared to controls $(\mathrm{p}<0.05)$. SFRP2 protein expression also tended to be more highly expressed in IPF arterioles $(p=0.10)$. These results corroborate the gene expression data.

\section{DISCUSSION}

Our findings provide direct evidence that genes involved in vascular proliferation and aberrant apoptosis are differentially expressed in pulmonary arterioles of IPF patients with or without $\mathrm{PH}$ compared with controls. Furthermore, patients with IPF who were free of $\mathrm{PH}$ and those with coexistent $\mathrm{PH}$ had similar gene expression in their pulmonary arterioles, 


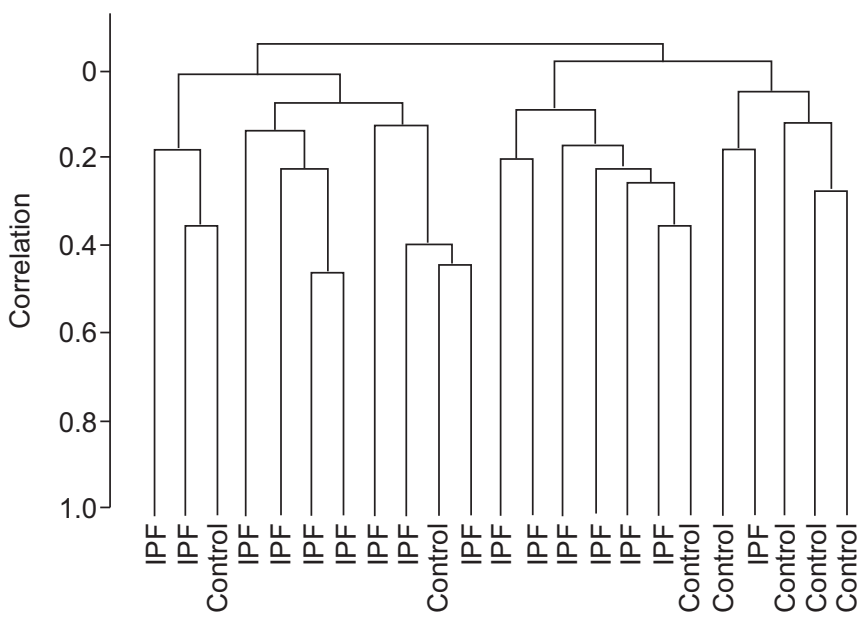

FIGURE 2. Idiopathic pulmonary fibrosis (IPF) vessels versus control vessels. Unsupervised hierarchical clustering analysis, using uncentered correlation and average linkage. The dendogram demonstrates clustering of gene expression of IPF samples separately from controls.

suggesting that pre-clinical pulmonary vascular disease may be present in IPF patients with normal haemodynamics.

The mechanisms that mediate pulmonary vascular remodelling in IPF are unknown. To our knowledge, this is the first study to utilise gene expression profiling of LCM pulmonary arterioles, a technically demanding process in fibrotic lung disease, to examine vascular gene expression in IPF. The patients in our study also underwent extensive and precise clinical phenotyping.

Two previous studies of gene expression profiling in PH-IPF have utilised whole-lung homogenates. GAGERMEIER et al. [5] reported gene expression data from 13 patients with $\mathrm{PH}-\mathrm{IPF}$. They found decreased expression of VEGF, endothelin (ET)-1 and platelet endothelial cell adhesion molecule, while phospholipase A2 and other inflammatory genes were upregulated. We also found that genes associated with VEGF signalling were downregulated, although inflammatory genes were not highly represented in our IPF samples. RAJKUMAR et al. [4] compared whole-lung gene expression from 18 subjects with $\mathrm{PAH}$ to that of eight subjects with PH-IPF. Rho-kinases and mediators of cAMP signalling were upregulated in $\mathrm{PAH}$ compared with PH-IPF. It is difficult to make a direct comparison between this study and ours, as we did not examine patients with PAH. More recently, MuRA et al. [12] examined lung gene expression in patients with varying aetiologies of pulmonary fibrosis (UIP and non-UIP) with and without severe $\mathrm{PH}(\mathrm{mPAP}>40 \mathrm{mmHg})$. Cellular proliferation and fibroblast migration pathways were highly expressed in the $\mathrm{PH}$ group. While the former pathway was also highly represented in the vasculature of IPF patients in our study, there was no overlap in the most highly upregulated genes. There were significant phenotypic differences between the patients in this study and ours. Differences in results may further be explained by our direct focus on isolated pulmonary arterioles rather than the mix of parenchyma, airways and vessels in prior studies. Investigation of isolated pulmonary arterioles enabled us to determine gene

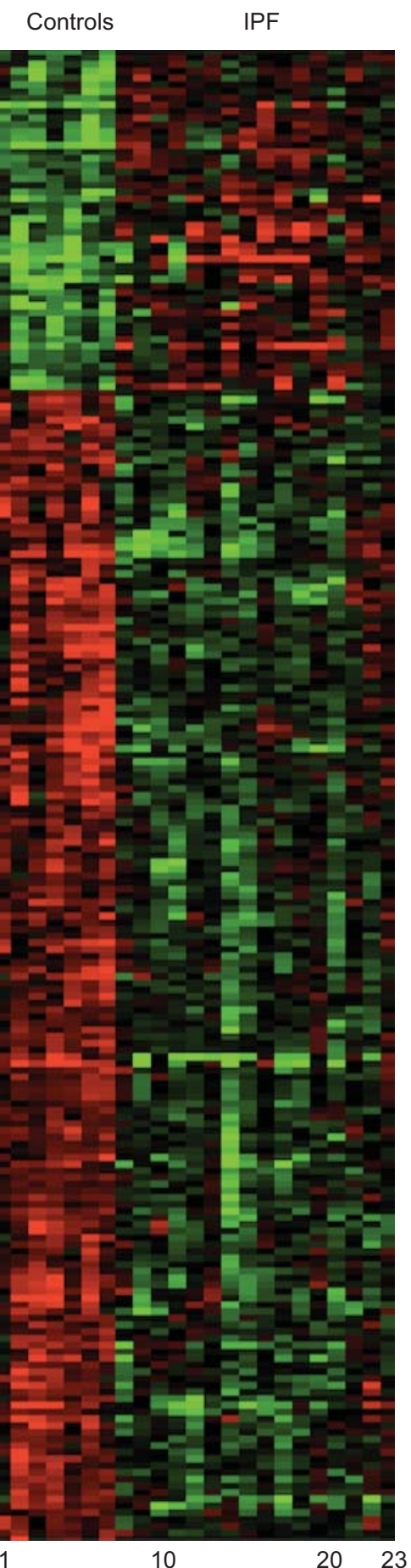

FIGURE 3. Heat map of differentially expressed genes in idiopathic pulmonary fibrosis (IPF) vessels versus controls. 259 genes differentially expressed at $\mathrm{p}<0.001$ with false discovery rate $<10 \%$. Red: upregulated genes; green: downregulated genes.

expression directly in the pulmonary vasculature, without potential artefacts of lung parenchyma affected by IPF.

Genes that promote cellular proliferation were upregulated in IPF pulmonary arterioles regardless of the presence of coexistent $\mathrm{PH}$. SMOC2 promotes angiogenesis and endothelial proliferation [13], and SMOC2 ablation inhibits DNA synthesis 


\begin{tabular}{|c|c|c|c|c|c|c|}
\hline Gene description & Gene symbol & Probe set ID & $\begin{array}{c}\text { Parametric } \\
\text { p-value }\end{array}$ & FDR & Fold-change & $\begin{array}{c}\text { Direction in } \\
\text { IPF }\end{array}$ \\
\hline \multicolumn{7}{|l|}{ Cellular proliferation } \\
\hline Signal transducer and activator of transcription 1, $91 \mathrm{kDa}$ & STAT1 & 200887_s_at & 0.0001192 & 0.03 & 1.8 & Up \\
\hline YY1 transcription factor & YY1 & 224718_at & 0.0003999 & 0.05 & 0.58 & Down \\
\hline Kruppel-like factor 10 & KLF10 & 202393_s_at & 0.0001628 & 0.03 & 0.44 & Down \\
\hline Transforming growth factor- $\beta$-induced, $68 \mathrm{kDa}$ & TGFBI & 201506_at & $1.41 \times 10^{-5}$ & 0.01 & 2.41 & Up \\
\hline Thy- 1 cell surface antigen & THY1 & 213869 x_at & 0.0001963 & 0.03 & 3.53 & Up \\
\hline \multicolumn{7}{|l|}{ Wnt signalling } \\
\hline Secreted frizzled-related protein 2 & SFRP2 & 223122_s_at & 0.0004054 & 0.05 & 9 & Up \\
\hline \multicolumn{7}{|l|}{ Apoptosis } \\
\hline \multicolumn{7}{|l|}{ Endothelial dysfunction/proliferation } \\
\hline Plasminogen activator, urokinase & PLAU & 205479_s_at & 0.0001612 & 0.03 & 2.29 & Up \\
\hline Sphingosine-1-phosphate receptor 1 & S1PR1 & 239401_at & 0.000134 & 0.03 & 0.45 & Down \\
\hline Kruppel-like factor 6 & KLF6 & 224606_at & $9.92 \times 10^{-5}$ & 0.03 & 0.53 & Down \\
\hline \multicolumn{7}{|l|}{ Hypoxia-related signalling } \\
\hline Arginase, type II & ARG2 & 203946_s_at & 0.000145 & 0.03 & 0.4 & Down \\
\hline Hypoxia inducible factor $3, \alpha$-subunit & HIF3A & 219319_at & 0.0006036 & 0.05 & 0.34 & Down \\
\hline Rho-GTPase activating protein 26 & ARHGAP26 & 205068_s_at & $8.69 \times 10^{-5}$ & 0.03 & 0.47 & Down \\
\hline \multicolumn{7}{|l|}{ Complement activation } \\
\hline Complement component 4A & C4A & 214428 x_at & $5.8 \times 10^{-6}$ & 0.01 & 2.93 & Up \\
\hline Complement component 4B & $\mathrm{C} 4 \mathrm{~B}$ & 208451_s_at & $2.07 \times 10^{-5}$ & 0.01 & 2.71 & Up \\
\hline
\end{tabular}

Univariate analysis demonstrated 255 differentially expressed genes between IPF arterioles and controls $p<0.001, q<10$. A full list is available in the online supplementary material. FDR: false discovery rate; SPARC: secreted protein, acidic, cysteine-rich; YY1: yin and yang protein; Thy-1: thymus cell antigen 1; Pim-1: proviral integration site 1; Rho-GTPase: Rho-guanosine triphosphate hydrolase.

in mouse fibroblasts exposed to PDGF- $\beta$ and other growth factors implicated in the pathogenesis of IPF [14]. Additionally, SMOC2 promotes cell cycle progression via cyclinD1, which was also upregulated in our samples [15]. Given these findings, it is plausible that SMOC2 contributes to vascular remodelling in IPF. In addition, gene and protein expression of STAT1 was increased in IPF pulmonary arterioles regardless of the presence of coexistent PH. STAT1 increases vascular

TABLE 3 Ingenuity pathways analysis of differentially expressed genes in idiopathic pulmonary fibrosis (IPF) arterioles versus controls

Cancer

Apoptosis and cell death

Jak-STAT signalling

Complement and coagulation cascade

Cellular growth and proliferation
ABAT, ADRA2C, AMD1, ARG2, ARHGAP26, ARIH2, C5, C4A, CAPN5, CCNL1, CFH, COL15A1, DUSP5, EIF1, HIF3A, HNRPDL, IGFBP3, INSR, KLF6, KLF10, LIFR, MAOA, MXRA5, PIM1, PLAU, SRFP2, STAT1, RIMP3

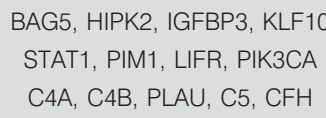

ANXA1, KLF10, PLAU, THY1, TGFBI, S1PR1, SMOC2, YY1 


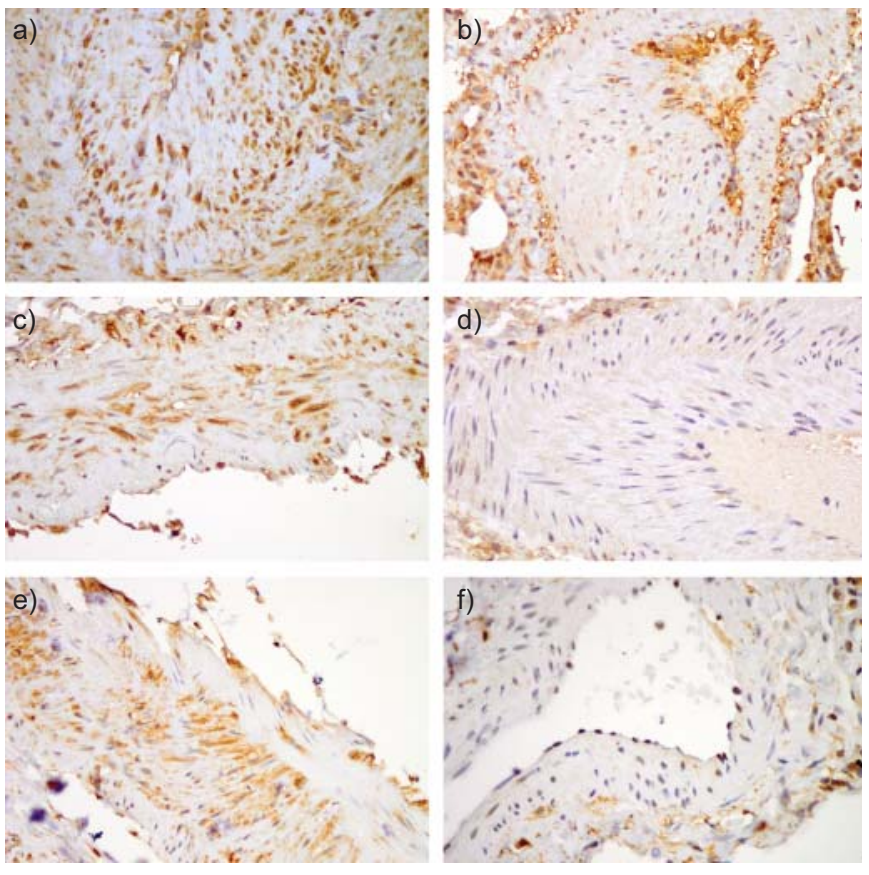

FIGURE 4. a) Blood vessel from usual interstitial pneumonia (UIP) tissue showing marked nuclear and cytoplasmic staining for signal transducer and activator of transcription 1 in endothelium and medial smooth muscle cells, while b) normal vessel shows only endothelial staining. c) blood vessel from UIP tissue shows intense cytoplasmic staining for secreted protein, acidic, cysteine-rich-related modular calcium binding (SMOC)-2 in medial smooth muscle cells and endothelial cells, while d) normal vessel is negative. e) blood vessel wall from UIP tissue shows cytoplasmic staining for secreted frizzled-related protein 2 in medial smooth muscle cells, but no staining in f) normal vessel. a, b) Magnification $\times 100, c-f$ ) magnification $\times 150$.

smooth muscle cell proliferation in response to angiotensin II and serotonin, proposed mediators of PAH [16, 17]. WoODS et al. [18] demonstrated that STAT1 mediates increased ET-1 production in human vascular smooth muscle cells (HVSMCs) [18]. Additionally, PLAU, which was overexpressed in our IPF samples, has been shown to activate STAT1 in both human vascular endothelial cells and HVSMCs [19, 20]. These data suggest that PLAU and other mediators may activate STAT1 in IPF, leading to vascular proliferation. Our finding of increased SFRP2 expression in IPF arterioles may indicate a role for Wnt signalling in promoting the development of PH-IPF. SFRP2, a mediator of both canonical and noncanonical Wnt signalling, promotes angiogenesis and inhibits hypoxia-induced endothelial cell apoptosis in experimental models [21]. Moreover, noncanonical Wnt signalling has recently been established to be active in the development of idiopathic PAH [22]. Lastly, genes that inhibit proliferation were downregulated in pulmonary arterioles of IPF patients with and without coexistent $\mathrm{PH}$. KLF10 mediates antiproliferative and antiapoptotic effects via TGF- $\beta$-Smad signalling [23]. KLF6 functions as a tumour suppressor which is expressed in endothelial cells, and is induced by hypoxia and other forms of cellular stress [24]. Downregulation of KLF10 and KLF6 may contribute to cellular proliferation and deregulation of apoptosis in the vasculature in IPF. YY1, a zinc-finger transcription factor that inhibits vascular smooth muscle cell growth and functions downstream from haem oxygenase (HO)-1, was downregulated in our samples
$[25,26]$. Reduced HO-1 expression characterises severe PAH, suggesting a potential association between the pathobiology of PAH and vascular change in IPF [26, 27]. Similar alterations in expression of genes that promote and inhibit cellular proliferation in pulmonary arterioles of IPF patients with and without coexistent $\mathrm{PH}$ strongly suggest that vascular function is altered early in the course of IPF. Such findings concur with previous reports indicating the development of clinical $\mathrm{PH}$ in the vast majority of IPF patients over time [2]. Alternatively, similar gene expression in pulmonary arterioles of IPF patients with or without $\mathrm{PH}$ may indicate that the biologic processes that account for pulmonary vascular changes in IPF may be a manifestation of IPF as a disease rather than $\mathrm{PH}$.

Interestingly, the genes that were differentially expressed in IPF vessels compared to controls largely fell into categories of cellular proliferation and cancer. Shared pathogenic ties between cancer biology and IPF, including aberrant cellular proliferation and impaired apoptosis, have been increasingly noted [28]. In PAH, STAT3 activation contributes to the propagation of hyperproliferative, apoptosis-resistant endothelial cells [29]. Several modulators of apoptosis were underexpressed in our samples. Conversely, mediators of proliferation, such as STAT1 and SMOC2, were upregulated $[19,20]$. These findings suggest that uncontrolled vascular proliferation and impaired apoptosis may contribute to vascular remodelling in IPF as well. Lastly, increased SFRP2 expression in our samples suggests an active role for Wnt signalling in the development of vascular changes in IPF [22].

There were several limitations in our study. We studied a small number of patients, albeit a typical sample size for gene microarray studies. We used LCM to isolate vessels for analysis, a technologically sophisticated approach that is novel in studies of the vasculature in IPF. Reverse transcriptase (RT)-PCR was not utilised for validation, as accurate dissection of pulmonary arteriolar tissue from architecturally distorted, fibrotic parenchyma precluded extraction of a sufficient quantity of tissue for both expression microarray analysis and RT-PCR. Patients with PH-IPF had only mild elevations in pulmonary artery pressure, but fulfilled the WHO criteria and are typical of PH-IPF $[3,30]$. Patients with more severe PH-IPF might have shown significant differences in gene expression compared to the NPH-IPF group. Combined pulmonary fibrosis with emphysema is associated with PH in IPF [31]; however, only one patient in our study had evidence of mild upper-lobe emphysema [31]. Moreover, spirometry and lung volumes were similar between the groups. We used specimens obtained from normal lung in patients undergoing carcinoid resection for a portion of control samples, and acknowledge that while the tissue appeared normal architecturally, it may have been abnormal at the molecular level. Lastly, while high-throughput gene expression analysis may be subject to Type I error, we constrained our false-positive rate and confirmed selected gene expression findings using immunohistochemistry.

In conclusion, the pulmonary vascular gene expression signature is similar in patients with coexistent IPF and $\mathrm{PH}$ and those with IPF alone and is characterised by aberrant cellular growth and deregulated apoptosis. Characterisation of pre-clinical pulmonary vascular alterations in IPF may lead to the development of novel therapeutic strategies for coexistent $\mathrm{PH}$ in these patients. 


\section{SUPPORT STATEMENT}

This work was supported by The Stony Wold-Herbert Fund (grant number CU08-7872).

\section{STATEMENT OF INTEREST}

Conflict of interest information can be found alongside the online version of this article at www.erj.ersjournals.com

\section{ACKNOWLEDGEMENTS}

We thank Neil Schluger (Chief, Division of Pulmonary, Allergy \& Critical Care Medicine, Columbia University, New York, NY) for his helpful comments after reviewing the manuscript.

\section{REFERENCES}

1 Patel NM, Lederer DJ, Borczuk AC, et al. Pulmonary hypertension in idiopathic pulmonary fibrosis. Chest 2007; 132: 998-1006.

2 Nathan SD, Shlobin OA, Ahmad S, et al. Serial development of pulmonary hypertension in patients with idiopathic pulmonary fibrosis. Respiration 2008; 76: 288-294.

3 Nathan SD, Shlobin OA, Ahmad S, et al. Pulmonary hypertension and pulmonary function testing in idiopathic pulmonary fibrosis. Chest 2007; 131: 657-663.

4 Rajkumar R, Konishi K, Richards TJ, et al. Genomewide RNA expression profiling in lung identifies distinct signatures in idiopathic pulmonary arterial hypertension and secondary pulmonary hypertension. Am J Physiol Heart Circ Physiol 2010; 298: H1235-H1248.

5 Gagermeier J, Dauber J, Yousem S, et al. Abnormal vascular phenotypes in patients with idiopathic pulmonary fibrosis and secondary pulmonary hypertension. Chest 2005; 128: Suppl. 6, $601 S$.

6 Patel NM, Kawut SM, Powell CA, et al. Gene expression profiling of pulmonary hypertension $(\mathrm{PH})$ in idiopathic pulmonary fibrosis (IPF). Am J Respir Crit Care Med 2008; 177.

7 Raghu G, Collard HR, Egan JJ, et al. An official ATS/ERS/JRS/ ALAT statement: idiopathic pulmonary fibrosis: evidence-based guidelines for diagnosis and management. Am J Respir Crit Care Med 2011; 183: 788-824.

8 American Thoracic Society. Idiopathic pulmonary fibrosis: diagnosis and treatment. International consensus statement. American thoracic society (ATS), and the European Respiratory Society (ERS). Am J Respir Crit Care Med 2000; 161: 646-664.

9 Badesch DB, Champion HC, Sanchez MA, et al. Diagnosis and assessment of pulmonary arterial hypertension. J Am Coll Cardiol 2009; 54: S55-S66.

10 Simonneau G, Robbins IM, Beghetti M, et al. Updated clinical classification of pulmonary hypertension. J Am Coll Cardiol 2009; 54: Suppl. 1, S43-S54.

11 Krotova K, Patel JM, Block ER, et al. Hypoxic upregulation of arginase II in human lung endothelial cells. Am J Physiol Cell Physiol 2010; 299: C1541-C1548.

12 Mura M, Anruka M, Yun Z, et al. Gene expression profiling in the lungs of patients with pulmonary hypertension associated with pulmonary fibrosis. Chest 2012; 141: 661-673.

13 Rocnik EF, Liu P, Sato K, et al. The novel SPARC family member SMOC-2 potentiates angiogenic growth factor activity. J Biol Chem 2006; 281: 22855-22864.

14 Ask K, Martin GE, Kolb M, et al. Targeting genes for treatment in idiopathic pulmonary fibrosis: challenges and opportunities, promises and pitfalls. Proc Am Thorac Soc 2006; 3: 389-393.
15 Liu P, Lu J, Cardoso WV, et al. The SPARC-related factor SMOC-2 promotes growth factor-induced cyclin D1 expression and DNA synthesis via integrin-linked kinase. Mol Biol Cell 2008; 19: 248-261.

16 Marrero MB, Schieffer B, Li B, et al. Role of Janus kinase/signal transducer and activator of transcription and mitogen-activated protein kinase cascades in angiotensin II- and platelet-derived growth factor-induced vascular smooth muscle cell proliferation. J Biol Chem 1997; 272: 24684-24690.

17 Banes AK, Shaw SM, Tawfik A, et al. Activation of the JAK/STAT pathway in vascular smooth muscle by serotonin. Am J Physiol Cell Physiol 2005; 288: C805-C812.

18 Woods M, Wood EG, Bardswell SC, et al. Role for nuclear factor- $\kappa \mathrm{B}$ and signal transducer and activator of transcription 1/interferon regulatory factor-1 in cytokine-induced endothelin-1 release in human vascular smooth muscle cells. Mol Pharmacol 2003; 64: 923-931.

19 Dumler I, Kopmann A, Weis A, et al. Urokinase activates the JAK/ STAT signal transduction pathway in human vascular endothelial cells. Arterioscler Thromb Vasc Biol 1999; 19: 290-297.

20 Dumler I, Kopmann A, Wagner K, et al. Urokinase induces activation and formation of STAT4 and STAT1-STAT2 complexes in human vascular smooth muscle cells. I Biol Chem 1999; 274: 24059-24065.

21 Courtwright A, Siamakpour-Reihani S, Arbiser JL, et al. Secreted frizzle-related protein 2 stimulates angiogenesis via a calcineurin/ NFAT signaling pathway. Cancer Res 2009; 69: 4621-4628.

22 Laumanns IP, Fink L, Wilhelm J, et al. The noncanonical WNT pathway is operative in idiopathic pulmonary arterial hypertension. Am J Respir Cell Mol Biol 2009; 40: 683-691.

23 Subramaniam M, Hawse JR, Johnsen SA, et al. Role of TIEG1 in biological processes and disease states. J Cell Biochem 2007; 102: 539-548.

24 Warke VG, Nambiar MP, Krishnan S, et al. Transcriptional activation of the human inducible nitric-oxide synthase promoter by Kruppel-like factor 6. J Biol Chem 2003; 278: 14812-14819.

25 Santiago FS, Lowe HC, Bobryshev YV, et al. Induction of the transcriptional repressor Yin Yang-1 by vascular cell injury. Autocrine/ paracrine role of endogenous fibroblast growth factor-2. J Biol Chem 2001; 276: 41143-41149.

26 Beck K, Wu BJ, Ni J, et al. Interplay between heme oxygenase-1 and the multifunctional transcription factor Yin Yang 1 in the inhibition of intimal hyperplasia. Circ Res 2010; 107: 1490-1497.

27 Achcar RO, Demura Y, Rai PR, et al. Loss of caveolin and heme oxygenase expression in severe pulmonary hypertension. Chest 2006; 129: 696-705.

28 Vancheri C, Failla M, Crimi N, et al. Idiopathic pulmonary fibrosis: a disease with similarities and links to cancer biology. Eur Respir J 2010; 35: 496-504.

29 Masri FA, Xu W, Comhair SA, et al. Hyperproliferative apoptosisresistant endothelial cells in idiopathic pulmonary arterial hypertension. Am J Physiol Lung Cell Mol Physiol 2007; 293: L548-L554.

30 Shorr AF, Wainright JL, Cors CS, et al. Pulmonary hypertension in patients with pulmonary fibrosis awaiting lung transplant. Eur Respir J 2007; 30: 715-721.

31 Cottin V, Le Pavec J, Prévot G, et al. Pulmonary hypertension in patients with combined pulmonary fibrosis and emphysema syndrome. Eur Respir J 2010; 35: 105-111. 